\title{
The reduction of saccadic latency by prior offset of the fixation point: An analysis of the gap effect
}

\author{
PATRICIA A. REUTER-LORENZ, HOWARD C. HUGHES, and ROBERT FENDRICH \\ Dartmouth College, Hanover, New Hampshire
}

\begin{abstract}
The latency to initiate a saccade (saccadic reaction time) to an eccentric target is reduced by extinguishing the fixation stimulus prior to the target onset. Various accounts have attributed this latency reduction (referred to as the gap effect) to facilitated sensory processing, oculomotor readiness, or attentional processes. Two experiments were performed to explore the relative contributions of these factors to the gap effect. Experiment 1 demonstrates that the reduction in saccadic reaction time (RT) produced by fixation point offset is additive with the effect of target luminance. Experiment 2 indicates that the gap effect is specific for saccades directed toward a peripheral target and does not influence saccades directed away from the target (i.e., antisaccades) or choice-manual RT. The results are consistent with an interpretation of the gap effect in terms of facilitated premotor processing in the superior colliculus.
\end{abstract}

The latency to initiate a saccade in response to an eccentric target is typically on the order of $180-250 \mathrm{msec}$ (Carpenter, 1977; Wheeless, Boynton, \& Cohen, 1966). Saccadic reaction times (RTs) can be substantially reduced, however, simply by extinguishing the fixation stimulus 200-300 msec prior to target onset (see, e.g., Fischer \& Ramsperger, 1984; Saslow, 1967). In addition to reducing the average latency of saccades, a temporal gap between fixation point offset and target onset (henceforth referred to as the gap condition) may produce a subpopulation of saccades with a modal latency of 120 msec (Fischer \& Ramsperger, 1984, 1986). These have been called "express saccades" (e.g., by Fischer, 1987; Fischer \& Boch, 1983; Fischer \& Breitmeyer, 1987). The latency reduction produced by fixation stimulus offsets have been variously attributed to facilitated sensory processing (see, e.g., Reulen, 1984a), oculomotor readiness (Kalesnykas \& Hallett, 1987; Saslow, 1967), or attentional factors (see, e.g., Fischer, 1987). In the present experiments, we attempted to clarify the basis of latency facilitation in the gap condition by examining the effects of target luminance and response requirements.

Fixation point offsets could conceivably exert their effect by altering visual sensitivity. It might be easier, for instance, to detect eccentric flashes in a blank field than in the presence of a fixation stimulus. This possibility is considered in Reulen's (1984a, 1984b) model, which attributes latency reduction in the gap condition to enhanced processing of the visual target. In this model, it is assumed that saccadic RT represents the linear sum of several seri-

This research was supported by Grants AFOSR-89-0437 and NINCDS 5 PO1-NS-17778 and by a faculty research award from Dartmouth College to H. C. Hughes. The authors thank Robin Plager and Joanna Morris for their assistance with data collection. Address requests for reprints to Patricia Reuter-Lorenz, Program in Cognitive Neuroscience, Pike House, Dartmouth Medical School, Hanover, NH 03756. ally organized processing stages. Following Grice's random threshold theory of response latency (Grice, 1968), a "sensory stage" is posited, in which neural responses to target onset accumulate until a threshold is reached. Subsequent events represent oculomotor programming and efferent processes. In this model, the accumulation rate is a direct function of signal intensity and is constant over time.

Reulen's (1984a, 1984b) model accounts for the reduction in latency in the gap condition by hypothesizing that fixation point offsets increase the rate at which the sensory activity accumulates. The main features of Reulen's model are portrayed in Figure 1, which depicts the accumulation of sensory activity over time for bright and dim flashes. Given a constant threshold, it is evident that bright targets will reach threshold faster than dim ones. This model also predicts that the latency difference between gap and overlap conditions should increase as target luminance decreases. We tested this possibility directly in Experiment $\mathbf{1}$ by measuring saccadic RT to high- and low-luminance targets presented with and without a central fixation stimulus.

\section{EXPERIMENT 1}

\section{Method}

Subjects. The subjects were 8 Dartmouth undergraduates and 2 of the authors (P.R.L. and H.C.H.). All subjects had normal vision or vision corrected by contact lenses.

Apparatus. Three computer-controlled red light-emitting diodes (LEDs) served as the saccade targets and the fixation stimulus. At the viewing distance of $57 \mathrm{~cm}$, each LED subtended a $0.5^{\circ}$ visual angle. The target LEDs were positioned $7.0^{\circ}$ to the right or left of the fixation light. The luminance of the fixation light was $0.8 \mathrm{~cd} / \mathrm{m}^{2}$. Target luminance was either $40 \mathrm{~cd} / \mathrm{m}^{2}$ (bright targets) or $0.4 \mathrm{~cd} / \mathrm{m}^{2}$ (dim targets). Luminance was controlled by varying the voltages applied to each lamp via digital-to-analog converters. The position of the left eye was monitored with the Eye-trac 200 infrared scleral reflection device, which has a resolution of $.25^{\circ}$ 


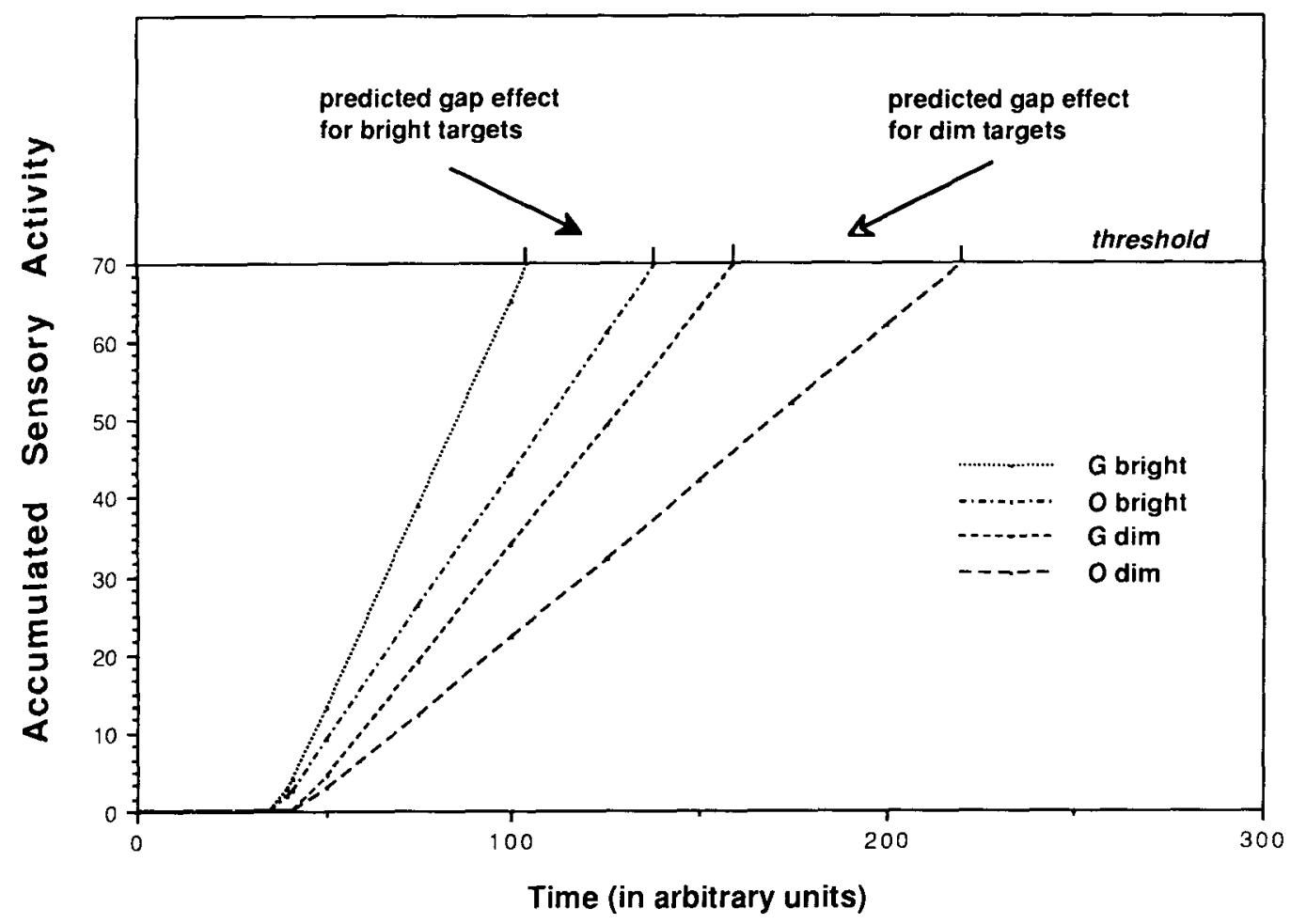

Figure 1. Diagram of Reulen's (1984a, 1984b) facilitation model. In this model, a sensory integrator accumulates activity following a peripheral afferent delay, which is a function of signal intensity. The rate of accumulation is jointly determined by signal intensity and a facilitation factor produced by fixation point offset. The facilitation factor, per se, is independent of target intensity. In this diagram, the parameters representing the facilitation factor and target intensity were multiplicatively combined, following Equation 1 of Reulen (1984a). Note that the time difference to reach threshold in the gap $(G)$ versus the overlap $(O)$ condition is larger for the dim than for the bright target. This interaction between the effects of intensity and fixation condition is predicted by the model regardless of whether the facilitation and intensity parameters are additively or multiplicatively combined.

and a 0 - to $250-\mathrm{Hz}$ bandwidth. The eye position signal was digitized at a sampling rate of $250 \mathrm{~Hz}$. Head movements were minimized by a chinrest/head-restraining assembly.

Design and Procedure. In this experiment, the effects of two levels of target luminance on saccadic RT were compared under the gap and overlap conditions. In the overlap condition, the fixation light was illuminated at the start and remained present throughout the trial. In the gap condition, the fixation point was illuminated at the start of the trial but was extinguished $200 \mathrm{msec}$ prior to the onset of the eccentric saccade target. Previous work has indicated that optimal gap effects can be obtained with a 200-msec gap interval (e.g., Fischer \& Breitmeyer, 1987; Saslow, 1967).

The procedure in Experiments 1 and 2 included several features designed to equate response readiness and minimize anticipatory responses. In previous investigations, fixation point offsets may have alerted the observer to the imminent occurrence of the visual target, perhaps resulting in responses faster than those in the overlap condition, in which no warning event occurred. In an effort to equate warning cues in the gap and overlap conditions, an auditory warning tone $(1000 \mathrm{~Hz})$ preceded the target on all trials. The warning tone was presented via a speaker positioned directly below the fixation LED. To minimize anticipatory responses, the positions of the target were unpredictable, and both fixation conditions included catch trials (20\%) in which no target was presented. Gap and overlap trials occurred randomly and with equal frequency within each block of 76 trials.
A schematic illustration of the events in each trial is presented in Figure 2. Each trial began with the illumination of the fixation light. After $900 \mathrm{msec}$, the 100 -msec warning tone was presented. In the gap condition, the fixation light was extinguished simultaneously with the offset of the warning tone, and, following a 200 msec dark interval, either the right or the left LED was illuminated for $300 \mathrm{msec}$. In the overlap condition, the target was presented $200 \mathrm{msec}$ after the offset of the warning tone, but the fixation light remained on until $500 \mathrm{msec}$ after target offset. Bright and dim targets were presented randomly within a trial block and occurred equally often in gap and overlap trials. The subjects were instructed to maintain central fixation until the onset of the eccentric target, at which point they were to look at the target as rapidly as possible.

Each subject participated in at least two practice blocks of 76 trials each, followed by six experimental blocks run over a 5-day period. No more than two trial blocks were run in each experimental session.

Data analysis. Saccades were detected automatically, with a velocity criterion of $>80 \mathrm{deg} / \mathrm{sec}$, but they were verified by the experimenters. The temporal interval between the onset of the target and the beginning of the saccade was taken as the saccadic latency. Following previous studies (e.g., Fischer \& Ramsperger, 1984, 1986), latencies less than $80 \mathrm{msec}$ were counted as anticipations. Latencies longer than 700 msec were considered misses and excluded from the analyses. Saccade magnitudes and peak velocities were computed with calibration data obtained immediately prior to each session. 


\section{OVERLAP CONDITION}

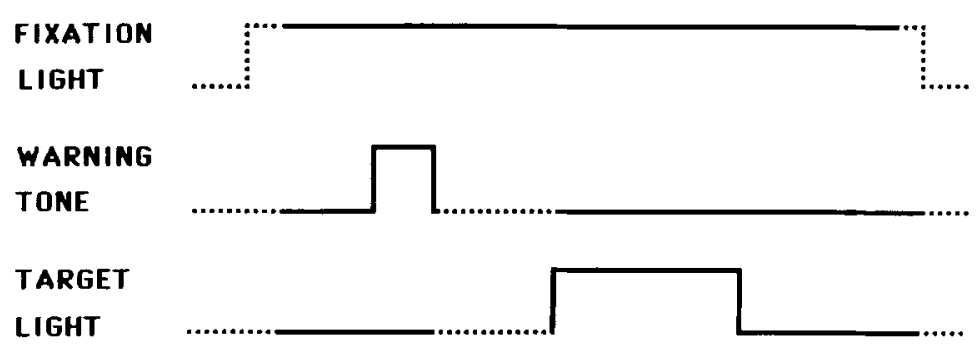

\section{GAP CONDITION}

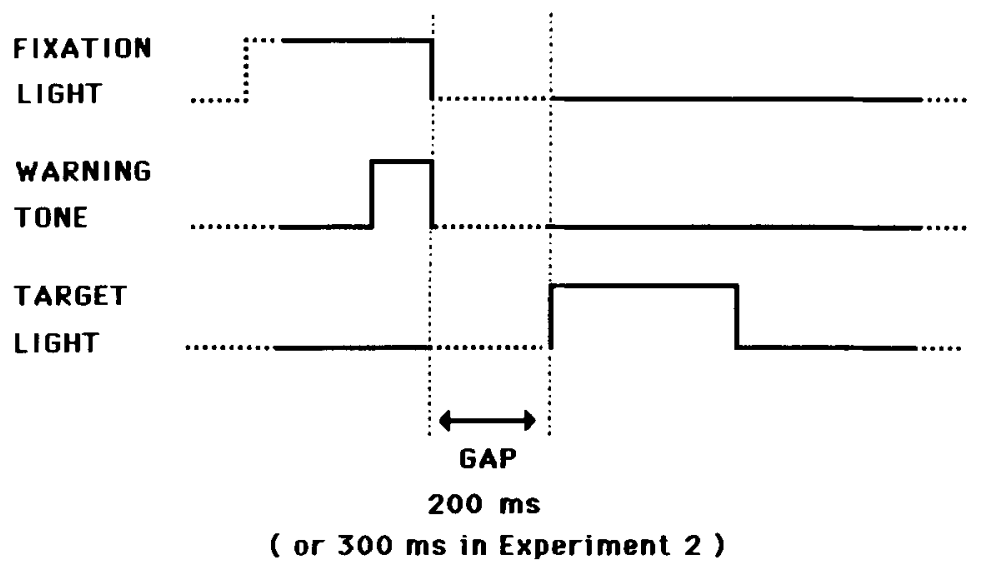

Figure 2. Schematic representation of trial events in gap and overlap conditions for Experiments 1 and 2.

\section{Results}

The latency data for bright and dim targets in the gap and overlap conditions are plotted in Figure 3. A twoway analysis of variance (ANOVA) on these data indicated significant main effects of fixation condition $[F(1,9)$ $=73.2, p<.0001]$ and intensity $[F(1,9)=472.1$, $p<.0001]$, but no significant interaction $[F(1,9)=$ $0.72]$. These results indicate a significant reduction of saccade latencies in the gap condition and demonstrate that this effect is additive with the facilitatory effect of target luminance. Together, fixation condition and target intensity accounted for $77 \%$ of the variance in the data set.

Response distributions. Since previous studies done with the gap paradigm have indicated that express saccades are often revealed as a prominent early mode in latency histograms, we obtained latency histograms for each subject in all conditions. The latency histograms for 2 representative subjects are shown in Figure 4. As was the case for all the subjects, the histograms show little evidence of bimodality. Given the differences in procedure used to collect the present data (i.e., the presence of catch trials, inclusion of an auditory warning signal, and position uncertainty), we tentatively attribute the absence of a bimodal latency distribution to procedural differences between this and previous work.

Anticipatory responses. Although anticipatory responses (i.e., latencies less than $80 \mathrm{msec}$ ) were rare, the mean incidence of anticipations was virtually identical in the overlap and gap conditions $(1.8 \%$ and $1.2 \%$, respectively). The similarity in these anticipation rates argues against the possibility that facilitation in the gap condition is due to a greater tendency to initiate responses prior to the target onset (Kalesnykas \& Hallett, 1987).

Saccade topology. Previous reports describing express saccades have provided little information about the topological aspects of saccades in the gap paradigm. We ob- 


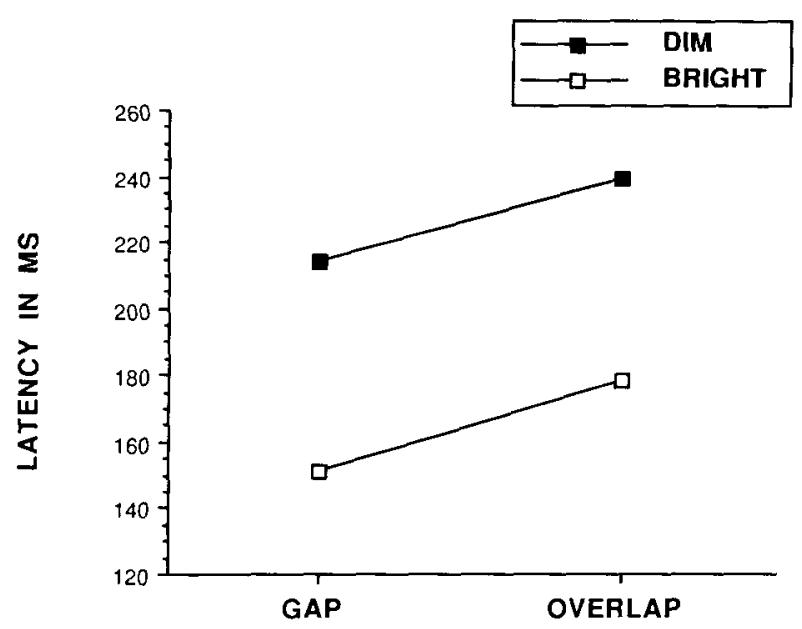

Figure 3. Mean saccade latencies to bright and dim targets in the gap and overlap conditions. served some variability in the amplitude (i.e., the size of the saccade, expressed in degrees) and velocity of saccades to the targets at $7.0^{\circ}$ and therefore analyzed these characteristics. An analysis of the amplitude data indicates only that saccades to the dim targets were slightly but significantly smaller than those to bright targets in both the gap and overlap conditions $[F(1,9)=16.4, p=.003]$. The average size of the saccades to dim targets at $7.0^{\circ}$ was $6.3^{\circ}$ versus $6.6^{\circ}$ in response to bright targets at the same eccentricity. An analysis of the peak velocities indicated no effect of luminance $[F(1,9)=0.6]$ or fixation condition $[F(1,9)=0.4]$.

\section{Discussion}

The bimodal latency distributions previously observed in the gap condition (e.g., by Fischer \& Ramsperger, 1984; Reulen, 1984b) were not found in the present experiment. These data suggest that bimodal distributions are not a necessary correlate of latency reduction in the

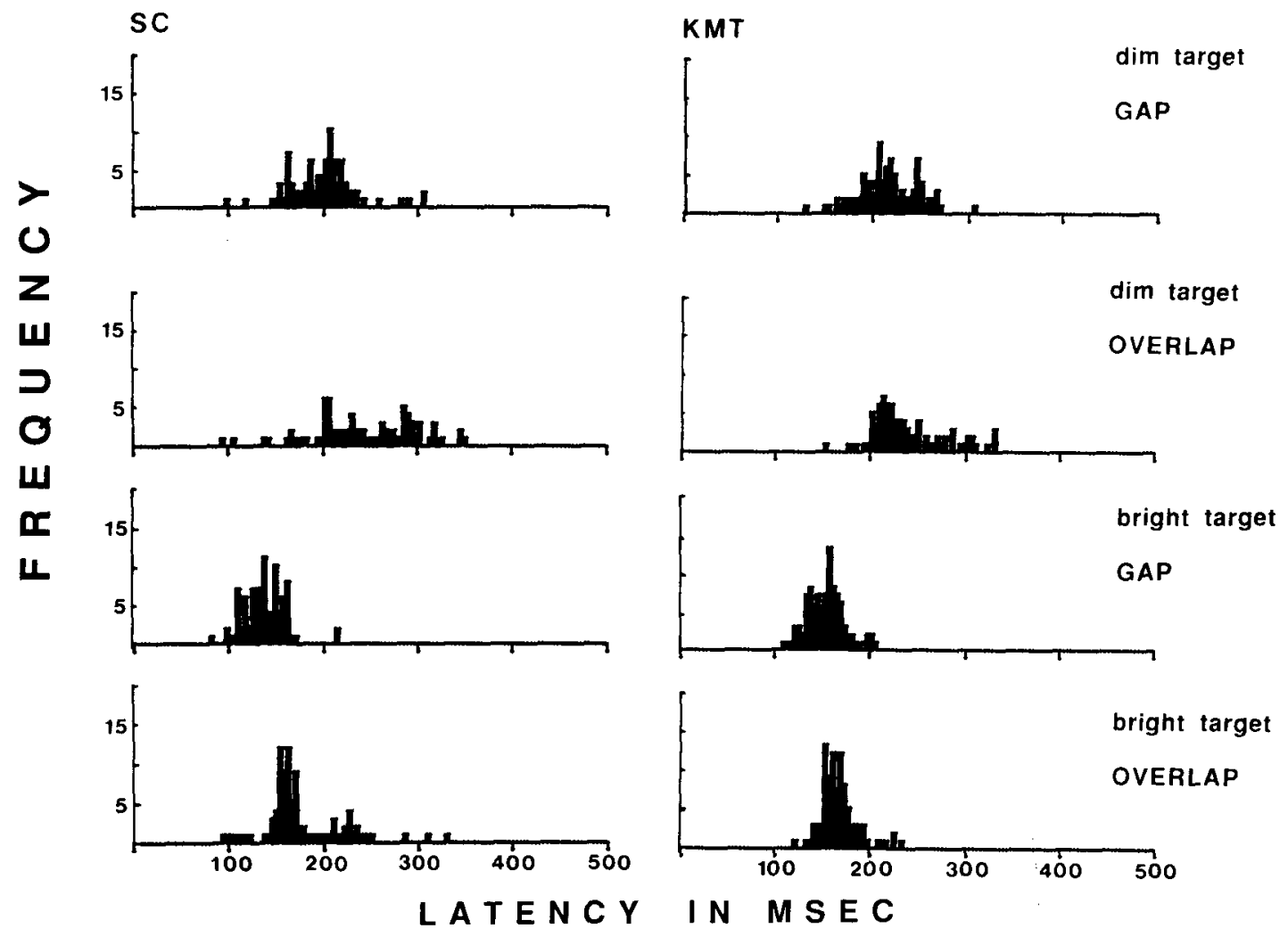

Figure 4. Frequency histograms representing the saccade latencies to bright and dim targets in the gap and overlap conditions for 2 observers (bin width $=8$ ). 
gap paradigm. In the bright-target condition, we observed many saccades with latencies within the range of 100-120 msec, in which the subpopulation of "express saccades" has been previously observed. However, none of our subjects showed evidence of a bimodal distribution, even though every one demonstrated a reduction in mean RT in the gap condition. Furthermore, the present results suggest that the facilitatory effects of fixation point offset do not depend on the absolute latency of the saccadic responses, since the magnitude of the gap effect was identical for bright and dim targets even though responses to dim targets were approximately $70 \mathrm{msec}$ slower. In addition, analyses of the topology of saccades generated in the gap condition indicate that they do not differ from "regular" saccades in accuracy or velocity.

The major finding in this experiment is that the reduction in saccadic RT produced by extinguishing the fixation light is equivalent for bright and dim targets. This additivity between the effects of luminance and fixation condition is incompatible with the mechanisms proposed by Reulen (1984a, 1984b) to account for the gap effect. By the logic of additive factors, this additivity raises the possibility that target luminance and fixation offsets exert their effects on different processing stages (Sternberg, 1969). Since the time course of neural activity is strongly dependent on stimulus intensity from the level of the photoreceptors to the primary visual cortex (see, e.g., Baylor \& Hodgkin, 1973; Lennie, 1981; Miller \& Glickstein, 1967 ), the present findings suggest that fixation point offsets might influence processes subsequent to the loci of intensity-dependent effects.

While additive factors logic has generated a coherent and internally consistent account of simple sensorimotor tasks (see, e.g., Sanders, 1977), it assumes serial, independent processing stages. This assumption has been challenged in some contexts, however (see, e.g., Eriksen \& Schultz, 1979; McClelland, 1979; Taylor, 1977). Thus, one goal in Experiment 2 was to test further the role of enhanced early sensory processing in mediating the gap effect.

\section{EXPERIMENT 2}

If fixation offsets produce enhanced sensory processing in the primary visual pathway, then a variety of responses might show reduced latencies in the gap condition. However, if the effect is restricted to saccades, it would suggest that fixation offsets specifically relate to processes within the oculomotor system. This possibility was tested in Experiment 2, in which the effects of fixation point offsets on manual RTs were compared with those on two different types of oculomotor responses: prosaccades, in which the saccade is directed to the target, and antisaccades, in which the saccades are directed away from the target (Hallett, 1978). In addition, by comparing pro- and antisaccades, we can evaluate whether the gap effect is a general characteristic of saccadic responses, a possibility suggested by several accounts of latency reduction in the gap paradigm (Fischer \& Breitmeyer, 1987; Kalesnykas \& Hallett, 1987; Saslow, 1967).

The account originally proposed by Saslow (1967) attributes the reduction of saccade latencies in the gap condition to the saccadic refractory period. Normal saccades and microsaccades are followed by a refractory period lasting approximately $150 \mathrm{msec}$, during which a second saccade cannot be initiated (Carpenter, 1977; Nachmias, 1959). Saslow reasoned that subjects are more likely to make microcorrective saccades when a fixation point is present than they are when it is absent. Thus, in the overlap condition, there is a greater likelihood that subjects are in the midst of the refractory period at the moment of target onset, which delays the saccadic response. According to this view, we might expect both pro- and antisaccades to be influenced by fixation point offsets, since the saccadic refractory period should affect both types of saccade equally. However, since this refractory period should not influence manual RTs, no effect of fixation offset would be expected in this condition.

More recently, Kalesnykas and Hallett (1987) have proposed that in the gap condition saccades may be programmed prior to the onset of the eccentric target. This view assumes that saccades are programmed without information about target location, so that the parameters of the motor program are initially set in the absence of visual input. According to this hypothesis, anticipatory saccades are initiated too far in advance of the target onset to be modified by its visual coordinates and therefore may be incorrect in direction and/or amplitude. Express saccades are thought to be preprogrammed in the appropriate direction by chance and are initiated late enough to have their amplitude modified by the target coordinates, resulting in short latencies and an accurate movement. Regular latency saccades are those that either are not preprogrammed or were reprogrammed in response to the target coordinates. Given that manual movements could also be preprogrammed, this view does not exclude the possibility that fixation offset could reduce choice-manual RTs. It does, however, predict that antisaccades should show a gap effect, since fixation offsets could trigger the proportion of preprogrammed saccades that happened to be in the direction opposite that of the target.

Finally, Experiment 2 is relevant to the proposal that attentional disengagement mediates saccadic facilitation in the gap condition (Fischer, 1987; Fischer \& Breitmeyer, 1987). According to this view, extinguishing the fixation point disengages attention. Thus, at the time of target onset, because attention is already disengaged, saccadic latencies are reduced by the amount of time normally required to disengage it. If attention must be disengaged prior to the occurrence of any saccade, as Fischer and Breitmeyer (1987) suggest, the latency of antisaccades might also be facilitated in the gap condition.

\section{Method}

Stimuli and Procedure. The stimulus display differed from that in Experiment 1 in that only the bright target $\left(40 \mathrm{~cd} / \mathrm{m}^{2}\right)$ was used. 
In addition, a 300-msec gap was included and randomly intermixed with 200-msec gap and overlap trials. On both gap and overlap trials, the 100 -msec warning tone offset either 200 or a $300 \mathrm{msec}$ prior to target onset and coincided with fixation point offset on gap trials. This means that, on overlap trials, there was either a 200 - or a 300 msec foreperiod between tone offset and target onset. On gap trials, this foreperiod corresponded to the 200 - or $300-\mathrm{msec}$ gap. In all other respects, the stimulus conditions were identical to those in Experiment 1.

Each subject participated in six trials blocks for each of three response conditions: prosaccade, antisaccade, and choice-manual RT. Two blocks of 76 trials each were run in each of nine experimental sessions carried out on separate days. A fixed order of response conditions was used, with all subjects participating in the prosaccade condition first, then the antisaccade condition, and finally the choice-manual RT task. In the antisaccade task, subjects were instructed to saccade the same distance but in the direction opposite the target light. In the choice-manual RT task, the subject pressed one of two response keys, depending on the location of the target. The stimulus-response mapping was always compatible (e.g., left light-left hand, right light-right hand). At least two blocks of practice trials preceded data collection for each response type. Manual RTs were accurate to the nearest millisecond.

Subjects. Three paid volunteers and 2 of the authors (P.R.L. and H.C.H.) participated in this experiment.

\section{Results}

The latency data are displayed in Figure 5. It is apparent that a strong gap effect emerged only for prosaccades. This was confirmed statistically in a three-way ANOVA with foreperiod (200 vs. $300 \mathrm{msec}$ ), fixation condition (gap vs. overlap), and response condition (manual, prosaccade, antisaccade) as repeated factors. In addition to main effects of response condition $[F(2,8)=16.4, p<.002]$ and fixation condition $[F(1,4)=23.4, p<.01]$, this analysis revealed a significant interaction between these factors $[F(2,8)=7.8, p<.02]$. Post hoc Newman-Keuls comparisons indicated that the interaction was

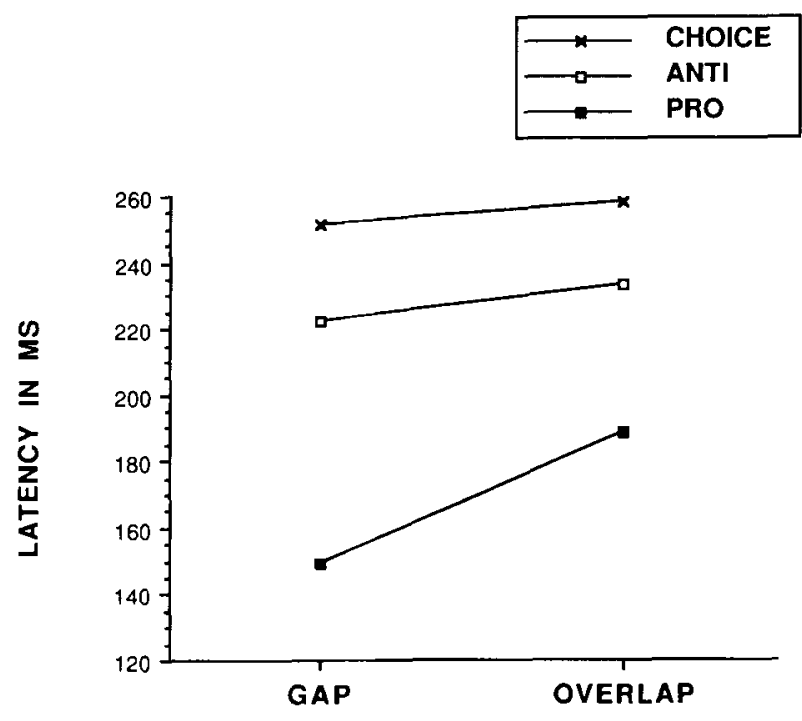

Figure 5. Mean latencies for the three response modes (prosaccades, antisaccades, and choice-manual responses) in the gap and overlap conditions. due to a significant latency reduction produced by fixation offset only for the prosaccade condition $(p<.05)$

The only other significant result was an interaction of foreperiod and response type $[F(2,8)=9.5, p<.01]$. The means indicate that, regardless of fixation condition, the 200-msec foreperiod was associated with faster responses for both pro- and antisaccades, whereas the 300 msec foreperiod produced slightly faster manual responses. Post hoc Newman-Keuls analyses indicated that the difference between foreperiods was significant only for antisaccades $(p<.05)$. The reasons for this foreperiod effect are not clear, but the pattern suggests differences in the time course of alerting processes for different response systems (L. E. Ross \& S. M. Ross, 1980; S. M. Ross \& L. E. Ross, 1981). In the gap condition, the two levels of foreperiod correspond to gap durations of 200 and $300 \mathrm{msec}$. The absence of an interaction between foreperiod and fixation condition indicates that gap duration had no reliable effect on RT.

Response distributions. Latency histograms from $2 \mathrm{ob}-$ servers for the prosaccade condition are presented in Figure 6. As in Experiment 1, the distributions showed little evidence for bimodality, REP being the lone exception.

Direction errors and anticipations. Although generally rare (less than $3 \%$ of all responses), direction errors were more common in the antisaccade condition than in either the prosaccade or the choice-manual condition. Since only two errors occurred in the manual condition, these data were not analyzed further. The proportion of direction errors occurring in the gap and overlap conditions for pro- and antisaccades were transformed (arc sine) and submitted to an ANOVA. This analysis indicated that direction errors were more frequent in the antisaccade task than in the prosaccade task $[F(1,4)=39.8, p=.004]$ and were also more frequent in the gap than in the overlap condition $[F(1,4)=14.5, p<.02]$. There was no interaction between these two variables.

The higher frequency of direction errors in the gap condition again raises the possibility that the shorter latency in this condition reflects a greater incidence of anticipatory responses. However, the incidence of direction errors was greater for antisaccades than for prosaccades, and there was no gap effect for antisaccades. Thus, the facilitatory effects of the gap cannot be explained by a greater tendency to make anticipatory responses. In addition, the frequency of anticipatory responses was found to be unaffected by fixation condition. In the pro- and antisaccade conditions, $1.7 \%$ of all responses had latencies of less than $80 \mathrm{msec}$, whereas none of the manual responses were anticipatory. Proportions of anticipatory responses were computed for each subject, arc-sine transformed and analyzed with a three-way ANOVA, with fixation condition, foreperiod, and response type (pro- vs. antisaccade) as factors. No main effects or interactions approached significance.

Multiple saccades. A further analysis of the antisaccade data revealed a strong tendency for 4 of the subjects 


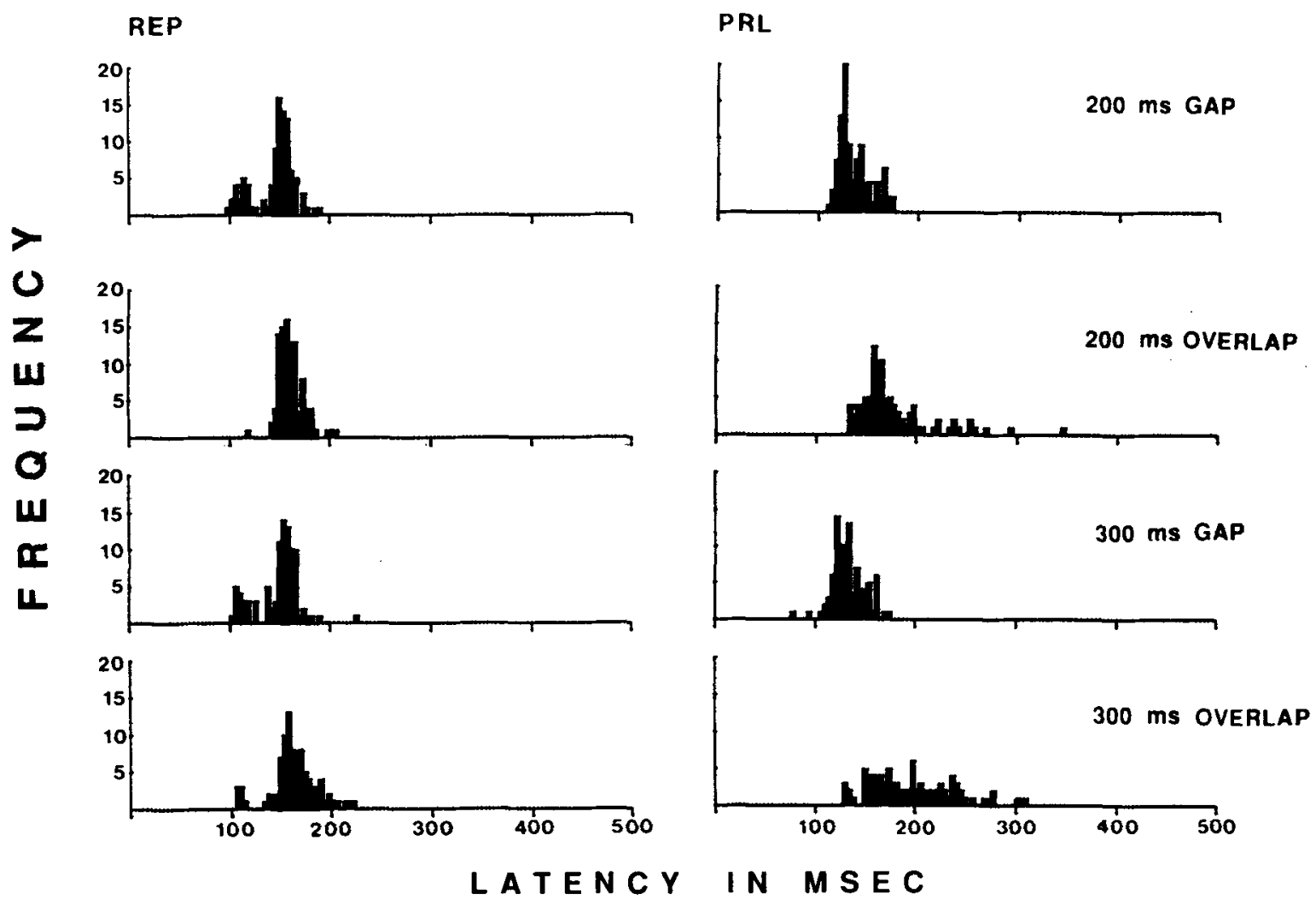

Figure 6. Frequency histograms representing the prosaccade latencies for 2 observers in the gap and overlap conditions with 200 and 300 msec foreperiods (bin width $=8$ ).

to generate "double saccades." For these subjects, $38 \%$ of the responses in this condition were characterized by primary and secondary saccades. In general, the magnitude of the secondary saccade was larger (greater than $60 \%$ of the primary) than is typical of corrective saccades (Carpenter, 1977). In his investigations of the antisaccade paradigm, Hallett (1978; Hallett \& Adams, 1980) also observed many multiple saccades in this task. Interestingly, Jay and Sparks (1990) report many double saccades in response to acoustic targets, suggesting that such saccadic responses may be a characteristic of saccades executed without visual guidance. Finally, it should be noted that the presence or absence of the fixation point had no effect on the frequency of double saccades.

\section{GENERAL DISCUSSION}

\section{Efferent Factors in the Gap Paradigm}

The results from Experiment 2 indicate that the facilitatory effect of fixation stimulus offset is limited to prosaccades. This response specificity, together with the finding that the gap effect is additive with the effect of target luminance, appears inconsistent with views that attribute the facilitatory effects of fixation point offsets to enhanced early visual processing. Both the additivity with target lu- minance and the response specificity are, however, consistent with the hypothesis that the gap effect may be related to premotor processes specifically within the oculomotor system.

Although the accounts of the gap effect offered by Saslow (1967) and Kalesnykas and Hallett (1987) also emphasize efferent factors, aspects of the present results are not readily explained by either view. If saccade direction and amplitude are preprogrammed in the gap condition, or if the saccadic refractory period contributes to the gap effect, we would expect antisaccade latencies to be facilitated by fixation point offset. The results from Experiment 2 provide no support for these expectations.

Other aspects of the data are inconsistent with the preprogramming hypothesis offered by Kalesnykas and Hallett (1987). In their view, the tendency to initiate preprogrammed saccades in the gap condition should produce more anticipatory saccades relative to the overlap condition. Using $80 \mathrm{msec}$ as a criterion for anticipations, we did not find this to be the case. Kalesnykas and Hallett propose that since anticipations tend to be hypometric, saccade amplitude should also be used to distinguish these responses from express saccades. We found no differences in saccade amplitude in the gap and overlap conditions, indicating that our data set does not in- 
clude substantial numbers of hypometric anticipatory responses.

Anticipatory factors may have played a greater role in some earlier work on the gap paradigm in which neither catch trials nor unpredictable target locations were used, or in which warning signals were not provided in both the gap and the overlap trials. In many previous studies (e.g., Fischer \& Ramsperger, 1986; Mayfrank, Mobashery, Kimmig, \& Fischer, 1986; Reulen, 1984a, 1984b), it is likely that, since the fixation point offset provided the only warning of the impending target event, subjects were more alert and prepared to respond in the gap condition than in the overlap condition (cf. L. E. Ross \& S. M. Ross, 1980; S. M. Ross \& L. E. Ross, 1981). The present data were obtained under conditions designed specifically to minimize the contribution of anticipatory processes. These measures appear to have successfully minimized anticipatory effects, as is evidenced by the similarity in anticipatory errors in the gap and overlap conditions.

\section{Role of the Superior Colliculus in Saccadic Facilitation}

Schiller, Sandell, and Maunsell (1987) report that ablations of the superior colliculus (SC) abolish "express" saccades, whereas they produce only a modest slowing of "regular" saccades. The close coupling of the sensory and motor fields in the SC and its direct output to brainstem oculomotor centers make it well suited for controlling the rapid foveation of eccentric targets (Sparks \& Mays, 1980; Wurtz \& Goldberg, 1972). Since observers are not normally compelled to fixate every peripheral event, some form of inhibitory control must be exerted on collicular mechanisms. Hikosaka and Wurtz (1983) have recently established a neural basis for such inhibition by demonstrating that activity in substantia nigra tonically inhibits the SC. Furthermore, electrophysiological observations indicate that the threshold current needed to elicit a saccade from either the SC or the frontal eye field (FEF) increases during active foveation (Goldberg, Bushnell, \& Bruce, 1986). Thus, it seems reasonable to suggest that the reduction in latency observed in the gap condition may reflect the functioning of collicular mechanisms in the absence of this fixation-related inhibition (i.e., fixation release). The absence of a gap effect for either choice-manual responses or antisaccades could be attributed to a lack of collicular involvement in either of these responses.

The FEFs have been implicated in the control of antisaccades, since patients with focal excisions involving this region are selectively impaired on this task (Guitton, Buchtel, \& Douglas, 1985). Signals originating in the FEF can control eye position independently of the SC (Schiller, True, \& Conway, 1980), perhaps via direct neuroanatomical connections to brainstem oculomotor centers (see, e.g., Leichnetz, 1981; Leichnetz, Smith, \& Spencer, 1984). To the extent that the FEFs can directly control saccades, responses requiring the FEF, such as antisaccades, may be independent of neural influences exerted on the colliculus. We suggest that a possible reason that antisaccades are not influenced by the gap condition is that their generation may be less dependent on the SC, where fixation point offsets appear to exert their effects. Given the highly voluntary nature of antisaccades, it is unlikely that special mechanisms would have evolved to inhibit their occurrence, as is the case for prosaccades.

The hypothesis that the gap effect reflects facilitated premotor processes that are specific to prosaccades suggests the possibility that the deeper layers of the SC might play an important role in this phenomenon, since many cells in this region are not visually responsive but show presaccadic activity (see, e.g., Jay \& Sparks, 1987). It would therefore be interesting to compare the activity of such presaccadic burst neurons in the gap and overlap conditions.

\section{Attentional Mediation of Latency Facilitation?}

Whereas we take the view that the latency facilitation in the gap condition reflects the release of oculomotor mechanisms from the inhibitory influences engaged during active fixation, Fischer and his colleagues attribute these effects to attentional disengagement (Fischer, 1987; Fischer \& Breitmeyer, 1987; Mayfrank et al., 1986). Several features of our data caution us against invoking attentional mechanisms to explain the gap effect. Considerable electrophysiological and behavioral evidence indicates that attention influences early stages of sensory processing (see, e.g., Hawkins, Shafto, \& Richardson, 1988; Mangun, Hillyard, \& Luck, in press). The work of Hawkins et al. (1988) is particularly relevant to the logic of the present investigation. They found that the magnitude of attentional effects produced by spatial precues interacted with target luminance, which led them to conclude that attention, like signal intensity, affected early visual processing (cf. Backus \& Sternberg, 1988). Furthermore, as Posner and his colleagues originally demonstrated (Posner, 1980; Posner, Nissen, \& Ogden, 1978), the facilitatory effects of attentional precues are not restricted to one response modality; they can enhance manual as well as saccadic RT. In contrast, the present results suggest that the gap effect operates quite differently from attentional manipulations in precuing paradigms, since it is additive with target luminance and is apparently restricted to prosaccades. Thus, if attentional mechanisms contribute to the gap effect, they differ from the mechanisms that mediate stimulus selection in spatial precuing paradigms and may be related instead to the selection of the appropriate oculomotor program (see Goldberg \& Seagraves, 1987).

\section{REFERENCES}

Backus, B. T., \& STeRnberg, S. (1988, November). Attentional tradeoff across space early in visual processing. Paper presented at the meeting of the Psychonomic Society, Chicago, IL. 
BAylor, D. A., Hodgkin, A. L. (1973). Detection and resolution of visual stimuli by turtle photoreceptors. Journal of Physiology, 206, 73-91.

Carpenter, R. H. S. (1977). Movements of the eyes. New York: Methuen.

ERiksen, C. W., Schultz, D. W. (1979). Information processing in visual search: A continuous flow conception and experimental results. Perception \& Psychophysics, 25, 249-263.

FISCHER, B. (1987). The preparation of visually guided saccades. Reviews of Physiology, Biochemistry and Pharmacology, 106, 1-35.

FisCheR, B., \& Boch, R. (1983). Saccadic eye movements after extremely short reaction times in the monkey. Experimental Brain Research, 260, 21-26.

FisCher, B., \& Breitmeyer, B. (1987). Mechanisms of visual attention revealed by saccadic eye motions. Neuropsychologia, 25, 73-83.

Fischer, B., \& RAMSPERGER, E. (1984). Human express saccades: Extremely short reaction times of goal directed eye movements. Experimental Brain Research, 57, 191-195.

FISCHER, B., \& RAMSPERGER, E. (1986). Human express-saccades: Effects of randomization and daily practice. Experimental Brain Research, 64, 569-578.

Goldberg, M. E., Bushnell, M. C., \& Bruce, C. J. (1986). The effect of attentive fixation on eye movements evoked by electrical stimulation of the frontal eye fields. Experimental Brain Research, 61, 579-584.

GoldberG, M. E., \& SEAGRaves, M. A. (1987). Visuospatial and motor attention in the monkey. Neuropsychologia, 25, 107-118.

Grice, R. G. (1968). Stimulus intensity and response evocation. Psychological Review, 75, 359-373.

Guitton, D., Buchtel, H. A., Douglas, R. M. (1985). Frontal lobe lesions in man cause difficulties in suppressing reflexive glances and in generating goal-directed saccades. Experimental Brain Research, $58,455-472$.

Hallett, P. E. (1978). Primary and secondary saccades to goals defined by instructions. Vision Research, 18, 1279-1296.

Hallett, P. E., \& ADAms, B. D. (1980). The predictability of saccadic latency in a novel voluntary oculomotor task. Vision Research, 20, 329-339.

Hawkins, H. L., Shafto, M. G., \& Richardson, K. (1988). Effects of target luminance and cue validity on the latency of visual detection. Perception \& Psychophysics, 44, 484-492.

Hikosaka, O., \& WURTz, R. H. (1983). Visual and oculomotor functions of monkey substantia nigra pars reticulata: I. Relation of visual and auditory responses to saccades. Journal of Neurophysiology, 49, 1230-1253.

JAY, M. F., \& SPARKs, D. L. (1987). Sensorimotor integration in the primate superior colliculus: II. Coordinates of auditory signals. Joumal of Neurophysiology, 57, 35-55.

JAY, M. F., \& SPARKS, D. L. (1990). Localization of auditory and visual targets for the initiation of saccadic eye movements. In M. A. Berkley \& W. Stebbins (Eds.), Comparative perception: Vol. 1. Basic mechanisms (pp. 351-374). New York: Wiley.

Kalesnykas, R., \& Hallett, P. E. (1987). The differentiation of visually guided and antícipatory saccades in gap and overlap paradigms. Experimental Brain Research, 68, 115-121.

LeichNETZ, G. R. (1981). The prefrontal cortico-oculomotor trajectories in the monkey: A possible explanation for the effects of stimulation/lesion experiments on eye movements. Journal of Neurological Sciences, 49, 387.

Leichnetz, G. R., Smith, D. J., \& Spencer, R. F. (1984). Cortical projections to the paramedian pontine tegmental and basilar pons in the monkey. Joumal of Comparative Neurology, 288, 388-408.

LENNIE, P. (1981). The physiological basis of variations in visual latency. Vision Research, 21, 815-824.

Mangun, G. R., HiLlyard, S. A., Luck, S. L. (in press). Elec- trocortical substrates of visual selective attention. In D. Meyer \& S. Kornblum (Eds.), Attention and performance IX. Hillsdale, NJ: Erlbaum.

Mayfrank, L., Mobashery, M., Kimmig, H., \& Fischer, B. (1986). The role of fixation and visual attention in the occurrence of express saccades in man. European Archives of Psychiatry \& Neurological Sciences, 235, 269-275.

MCCLELLAND, J. L. (1979). On the time relations of mental processes: An examination of systems of processes in cascade. Psychological Review, 86, 287-330.

Miller, J. M., \& Glickstein, M. (1967). Neural circuits involved in visuomotor reaction time in the monkey. Joumal of Neurophysiology, 30, 399-414.

NACHMIAS, J. (1959). Two dimensional motion of the retinal image during monocular fixation. Journal of the Optical Society American, 49, 901-908.

Posner, M. I. (1980). Orienting of attention. Quarterly Journal of Experimental Psychology, 32, 3-25.

Posner, M. I., Nissen, M. J., Ogden, W. C. (1978). Attended and unattended processing modes: The role of set for spatial location. In H. L. Pick \& I. J. Saltzman (Eds.), Models of perceiving and information processing. Hillsdale, NJ: Erlbaum.

Reulen, J. P. H. (1984a). Latency of visually evoked saccadic eye movements: I. Saccadic latency and the facilitation model. Biological Cybernetics, 50, 251-263.

REULEN, J. P. H. (1984b). Latency of visually evoked saccadic eye movements: II. Temporal properties of the facilitation mechanism. Biological Cybernetics, 50, 263-271.

Ross, L. E., \&oss, S. M. (1980). Saccade latency and warning signals: Stimulus onset, offset, and change as warning events. Perception \& Psychophysics, 27, 251-257.

Ross, S. M., \& Ross, L. E. (1981). Saccade latency and warning signals: Effects of auditory and visual stimulus onset and offset. Perception \& Psychophysics, 29, 429-437.

SANDERS, A. F. (1977). Structural and functional aspects of the reaction process. In S. Domic (Ed.), Attention and performance VI (pp. 325). Hillsdale, NJ: Erlbaum.

SASLOW, M. G. (1967). Effects of components of displacement-step stimuli upon latency of saccadic eye movement. Journal of the Optical Society of America, 57, 1024-1029.

SChiller, P. H., SANdell, J. H. \& Maunsell, J. H. R. (1987). The effect of frontal eye field and superior colliculus lesions on saccadic latencies in the rhesus monkey. Journal of Neurophysiology, 57, 1033-1049.

Schiller, P. H., True, S. D., \& Conway, J. L. (1980). Deficits in eye movements following frontal eye-field and superior colliculus ablations. Journal of Physiology, 44, 1175-1189.

SPARKS, D. L., \& MAYS, L. E. (1980). Movement fields of saccaderelated burst neurons in the monkey superior colliculus. Brain Research, 190, 39-50.

STERNBERG, S. (1969). The discovery of processing stages: Extensions of Donders' method. In W. G. Koster (Ed.), Attention and performance II (pp. 276-315). Amsterdam: North-Holland.

TAYLOR, D. A. (1977). Time course of context effects. Journal of Experimental Psychology: General, 106, 404-426.

Wheeless, L. L., Boynton, R. M., Cohen, G. H. (1966). Eye movement responses to step and pulse-step stimuli. Joumal of the Optical Society of America, 57, 396-400.

WURTZ, R. H., GoldBERG, M. E. (1972). Activity of superior colliculus in behaving monkey: IIl. Cells discharging before eye movements. Journal of Neurophysiology, 35, 575-586.

(Manuscript received February 26, 1990; revision accepted for publication August 30, 1990.) 